\title{
A longitudinal study investigating patient acquisition of azole resistant Aspergillus fumigatus (ARAf)
}

\author{
Amelie P Brackin ${ }^{* a}$, Anand Shah ${ }^{\mathrm{b}}$, Darius Armstrong-James ${ }^{\mathrm{a}}$ \\ ${ }^{a}$ Department of Infectious Diseases, Imperial College, London, UK \\ ${ }^{b}$ School of Public Health, Imperial College, London, UK
}

\begin{abstract}
The global emergence of azole resistance in Aspergillus fumigatus is resulting in health and food security concerns. Rapid diagnostics and environmental surveillance methods are key to understanding the distribution and prevalence of azole resistance in patients with chronic respiratory disease. This study will employ molecular methodologies to investigate the evolution of azole resistance during antifungal therapy and environmental surveillance of health care settings and patient homes will identify potential 'hot-spots' of ARAf.
\end{abstract}

Peer-review under the responsibility of the organizing committee of the ICMB21.

Keywords: Aspergillus fumigatus; azole resistance; environmental surveillance; chronic respiratory disease

\section{Introduction/Background}

The global burden of human associated mycoses is increasing. Each year, fungi account for billions of human infections and claim over 1.5 million lives worldwide ${ }^{1,2}$. Such fungal invasions incur considerable economic burden on the health care system which are likely underestimated due to inadequate diagnostics and lack of mycological surveillance ${ }^{2}$. Aspergillus fumigatus is a globally distributed saprophytic fungus found in soil, water and the air we breathe. Its vast geographical range has been facilitated by the rapid dispersion of airborne conidia that are able to tolerate a wide range of biotic stresses ${ }^{3,4}$. A. fumigatus is the primary etiological agent of a spectrum of diseases collectively known as aspergillosis, which depending on the hosts immune response can range from mild allergenic symptoms to severely life-threatening invasive aspergillosis (IA). Patients suffering from chronic infections include directly related diseases, such as allergic bronchopulmonary aspergillosis (ABPA) and chronic pulmonary aspergillosis (CPA), together with secondary conditions, such as cystic fibrosis (CF) and asthma. Currently, a limited selection of drugs are available for the treatment and prophylaxis of aspergillosis with oral triazole antifungals currently forming a lead role in the defense against the disease. However, continual exposure to antifungal compounds exerts selective pressures that can facilitate the evolution of resistance. Recently, there has been rapid global emergence of azole resistant Aspergillus fumigatus (ARAf) phenotypes, often conferred by the presence of genetic mutations in lanosterol 14- $\alpha$ demethylase (CYP51A), a component of the ergosterol biosynthetic pathway and target for azole antifungals ${ }^{5}$. Azole resistance can develop during patient therapy or within organic matter containing fungicide residues ${ }^{6,7}$. Some patients with chronic respiratory disease have never been exposed to antifungal therapy yet are infected with A. fumigatus carrying pre-adapted antifungal resistance indicating an environmental route of transmission ${ }^{8}$. The widespread use of azoles as agricultural fungicides coupled with the increasing reports of environmentally acquired resistant strains indicates that agricultural fungicide usage is escalating the incidence of azole-resistant infections in the clinic. In northern Europe, the prevalence of ARAf in IA has significantly increased from negligible levels pre-1999 to current rates of $\sim 25 \%{ }^{9,10}$. Moreover, mortality rates in patients with azole-resistant IA have been reported between $50 \%$ and $100 \%{ }^{11-13}$. Additionally, It has recently been reported that individuals with chronic respiratory diseases show a high prevalence of ARAf at $13 \%{ }^{14}$. Despite these dramatic figures, fungal pathogen research initiatives and resource investment is heavily neglected and underfunded compared to other infectious diseases ${ }^{15}$. Environmental surveillance and resistance monitoring are needed to fully appreciate the prevalence and distribution of ARAf isolates in the environment and will inevitably aid outbreak recognition, response and prevention ${ }^{15}$. The rapid emergence of ARAf and high prevalence in chronic respiratory disease patients has raised concerns that the medical azoles may cease to be effective in the future. Consequently, there

\footnotetext{
* Corresponding author. a.brackin@imperial.ac.uk
} 


\section{ICMB21}

is an urgent need to understand the evolutionary dynamics of azole resistant development and evaluate the acquisition of ARAf isolates in patients with chronic respiratory diseases.

\subsection{Aims}

The overarching aim of this project is to investigate the extent that patients are acquiring azole resistant infections from environmental sources, i.e. nosocomial, home or patient-patient transmission. Additionally, we will explore the evolution of drug resistance in chronic respiratory diseases.

\subsection{Methodology}

Through a longitudinal multicenter survey, sputum samples from $\sim 150$ adult patients diagnosed with chronic lung disease will be collected over an 18-month period. Azole susceptibility will be determined using clinical breakpoints (EUCAST testing) followed by a whole genome sequencing (WGS) approach to explore phylogenetic relationships and diversification of azole resistant mutations over time. Fine scale surveillance of nosocomial and patient home environments will be carried out for the duration of the longitudinal survey. ARAf strains isolated during the surveillance study will be WGS and analysed alongside patient samples to determine patient ARAf acquisition. Detection of clonal isolates within the sample population will enable identification of potential environmental 'hot-spots' of azole resistance. Isolates of interest will be phenotypically characterised using the high through-put Omnilog platform to investigate variations in metabolic profiles.

\subsection{Results}

The rate of azole resistance in isolates collected from environmental samples at the Royal Brompton Hospital, London currently stands at $\sim 4 \%$. However, as this study is still ongoing this is subject to change while environmental surveillance is expanded throughout the hospital. These findings highlight the risk ARAf poses to immunocompromised patients and is of particular concern during the COVID-19 pandemic.

\section{References}

1. Bongomin, F., Gago, S., Oladele, R. O. \& Denning, D. W. Global and multi-national prevalence of fungal diseasesestimate precision. Journal of Fungi vol. 3 (2017).

2. $\quad$ Brown, G. D. et al. Hidden killers: Human fungal infections. Science Translational Medicine (2012)

3. Pringle, A. et al. Cryptic speciation in the cosmopolitan and clonal human pathogenic fungus Aspergillus fumigatus. Evolution (N. Y). 59, 1886-1899 (2005).

4. Sewell, T. R. et al. Nonrandom distribution of azole resistance across the global population of Aspergillus fumigatus. MBio 10, (2019).

5. Parker, J. E. et al. Resistance to antifungals that target CYP51. Journal of Chemical Biology (2014)

6. Verweij, P. E., Snelders, E., Kema, G. H., Mellado, E. \& Melchers, W. J. Azole resistance in Aspergillus fumigatus: a side-effect of environmental fungicide use? The Lancet Infectious Diseases vol. 9 789-795 (2009).

7. Van Paassen, J., Russcher, A., In’t Veld-Van Wingerden, A. W., Verweij, P. E. \& Kuijper, E. J. Emerging aspergillosis by azole-resistant Aspergillus fumigatus at an intensive care unit in the Netherlands, 2010 to 2013. Eurosurveillance (2016)

8. Dauchy, C. et al. Emergence of Aspergillus fumigatus azole resistance in azole-naïve patients with chronic obstructive pulmonary disease and their homes. Indoor Air (2018)

9. Howard, S. J. et al. Frequency and evolution of azole resistance in Aspergillus fumigatus associated with treatment failure. Emerg. Infect. Dis. (2009)

10. Engel, T. G. P. et al. Prevalence and diversity of filamentous fungi in the airways of cystic fibrosis patients - A Dutch, multicentre study. J. Cyst. Fibros. (2019)

11. Snelders, E. et al. Emergence of azole resistance in Aspergillus fumigatus and spread of a single resistance mechanism. PLoS Med. (2008)

12. Chowdhary, A., Sharma, C. \& Meis, J. F. Azole-resistant aspergillosis: Epidemiology, molecular mechanisms, and treatment. J. Infect. Dis. (2017)

13. van der Linden, J. W. M. et al. Clinical implications of azole resistance in Aspergillus fumigatus, The Netherlands, 2007-2009. Emerg. Infect. Dis. (2011)

14. Abdolrasouli, A. et al. High prevalence of triazole resistance in clinical Aspergillus fumigatus isolates in a specialist cardiothoracic centre. Int. J. Antimicrob. Agents 52, 637-642 (2018).

15. Fisher, M. C., Hawkins, N. J., Sanglard, D. \& Gurr, S. J. Worldwide emergence of resistance to antifungal drugs challenges human health and food security. Science vol. 360 739-742 (2018). 